\title{
Regression in local recurrence in the contralateral breast following mastectomy in bilateral locally advanced breast cancer: A comparison of neutrophil-to-lymphocyte and platelet-to-lymphocyte ratios
}

\author{
Abdullah Durhan ${ }^{1}$ (D), Gamze Durhan² ${ }^{2}$, Aydan Eroğlu
}

ABSTRACT

ORCID IDs of the authors: A.D. 0000-0002-5622-9678; G.D. 0000-0002-6281-9287.

\section{Cite this paper as:} Durhan A, Durhan G, Eroğlu A. Regression in local recurrence in the contralateral breast following mastectomy in bilateral locally advanced breast cancer: a comparison of neutrophil-to-lymphocyte and platelet-to-lymphocyte ratios. Turk J Surg 2018; 34: 140-142.

'Department of General Surgery and Surgical Oncology, Ankara University School of Medicine, Ankara, Turkey

2Department of Radiology, Ankara Training and Research Hospital, Ankara, Turkey

\section{Corresponding Author} Abdullah Durhan e-mail:durhanabdullah@gmail.com

Received: 23.05.2015 Accepted: 04.08.2015 Available Online Date: 03.01.2018

\section{CCopyright 2018} by Turkish Surgical Association
The neutrophil-to-lymphocyte ratio is clinically accepted as a marker of systemic inflammatory response. In breast cancer patients, neutrophil-to-lymphocyte ratio can be used as an important prognostic indicator of survival. In routine laboratory tests, the platelet-to-lymphocyte ratio can also be examined in addition to neutrophil-to-lymphocyte ratio. Although the effects on breast cancer survival of platelet-to-lymphocyte ratio, which is accepted as the twin of neutrophil-to-lymphocyte ratio, are not as widely accepted as those of neutrophil-to-lymphocyte ratio, platelet activation is known to be a feature of cancer. Here, we present the neutrophil-to-lymphocyte ratio and platelet-tolymphocyte ratio of a patient with locally advanced cancer of the left breast who underwent a simple mastectomy that reduced the tumor load. Following surgical therapy, a remarkable regression was observed in the local recurrence area of the right mastectomy site; at the same time, the patient's neutrophil-to-lymphocyte ratio and PLR values significantly decreased.

Keywords: Locally advanced stage, breast cancer, simple mastectomy, neutrophil-to-lymphocyte ratio, platelet-tolymphocyte ratio

\section{INTRODUCTION}

Breast cancer is the most commonly observed type of cancer in women; in approximately $25 \%-30 \%$ of patients, diagnosis is made when the breast cancer is locally advanced (1). As in other cancers, the inflammatory response in breast cancer plays an important role in cancer development. The neutrophilto-lymphocyte ratio (NLR) and platelet-to-lymphocyte ratio (PLR), which can be obtained from a peripheral full blood count, are routinely clinically accepted as markers of systemic inflammatory response $(2,3)$. Recent studies have shown that the immune response increases with a reduction in tumor load (4). Here, we present the NLR and PLR of a patient with locally advanced breast cancer who underwent simple mastectomy that reduced the tumor load.

\section{CASE PRESENTATION}

A 52-year-old woman was referred to our clinic for surgical treatment of locally advanced breast cancer. From the patient's history, it was learned that one year previously, she had presented at another center with the complaint of a mass causing color change and rash bilaterally on the breasts. The patient was diagnosed with Grade 3 invasive ductal carcinoma breast cancer on the right side. Estrogen receptor (ER) and progesterone receptor (PR) tests were strongly positive, and HER2 expression was negative (luminal A tumor). She was administered eight cycles of neoadjuvant chemotherapy, and simple mastectomy was performed on the right breast followed by hormonotherapy. It is not known why the patient underwent mastectomy of only the right breast instead of a bilateral mastectomy at the other hospital. She applied to our clinic due to increased symptoms in her left breast. When the patient presented at our clinic, the mass in her left breast had grown, there was increased rash on the breast skin, and a new mass had developed on the incision scar of the right side of the mastectomy area.

In the physical examination, multiple nodular metastatic masses, the largest of which was $1 \mathrm{~cm}$ in diameter, were detected on the incision scar of the mastectomy site of the right breast along with fixed lymphadenopathies in the right axilla. The left breast was completely full of tumoral mass, with tumor invasions of the skin. Inflammatory appearance of the skin, satellite nodules, and fixed lymphadenopathies in the left axilla were observed. The preoperative images are shown in Figure 1.

No abnormal findings were seen in the PA pulmonary radiograph, abdominal pelvic ultrasonograph (USG), or bone scintigraph. No pathology was determined by routine biochemistry. The patient was informed, and a simple mastectomy was applied to the left breast to reduce the tumor load. As the skin was completely covered with the tumor, the skin borders close to the sternum could not be approached with the primary incision; after removal of the breast, which was infected and had tumor necrosis, sec- 


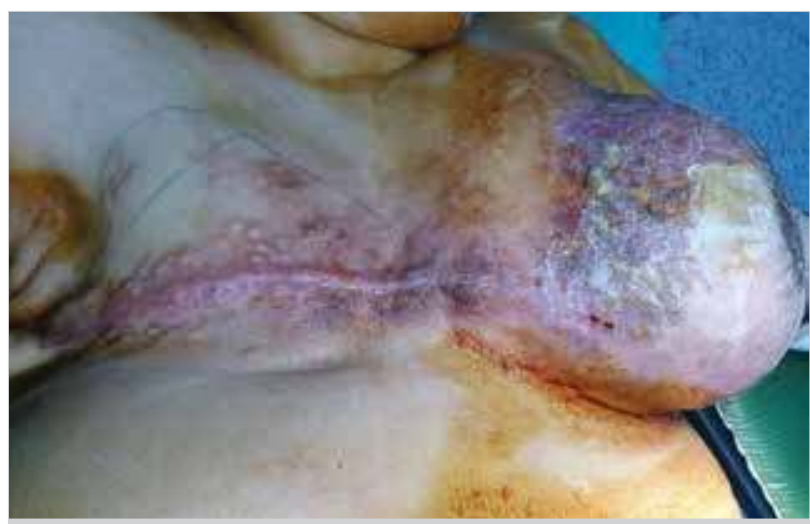

Figure 1. Preoperative image. The left breast was completely full of tumoral mass, with tumor invasions of the skin. An inflammatory appearance of the skin and satellite nodules were observed

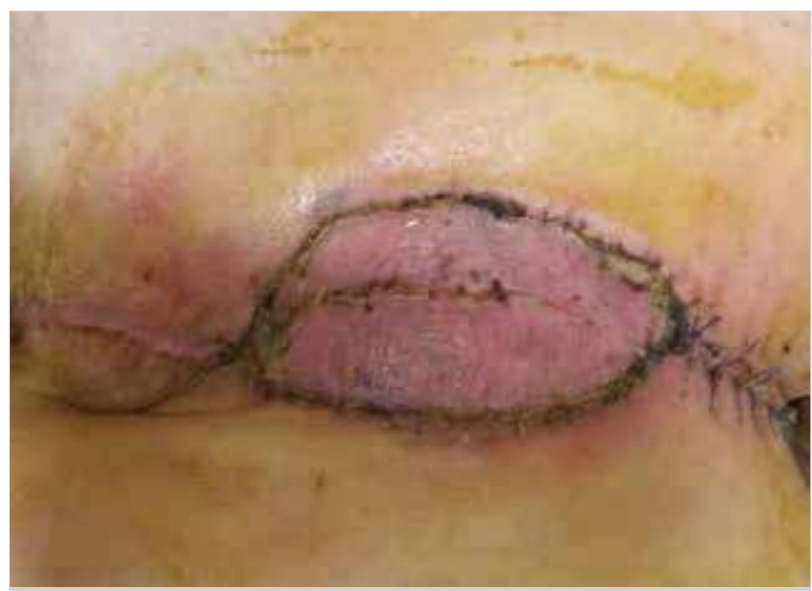

Figure 2. The healed left side graft area and the regression of local recurrences on the right side

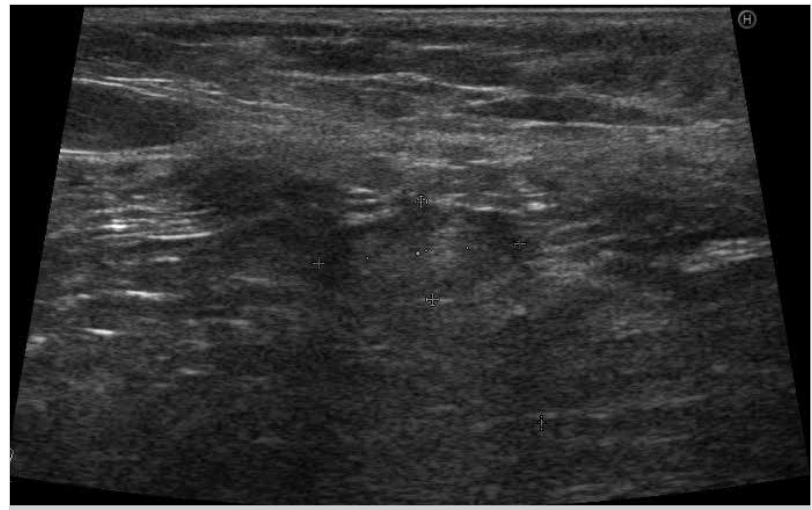

Figure 3. Axillary lymph node with thin cortex and hyperechogenic fatty hilus was demonstrated in ultrasound images

ondary healing was applied. After 10 days, as the granulation tissue was seen to be sufficient, the open wound was repaired with graft application. No postoperative complications developed, and the patient was discharged on postoperative day 7. After removal of the tumor load in the left breast, a regression in local recurrence sites in the right mastectomy area was observed. The healed left side graft area and the regression of local recurrences on the right side are shown in Figure 2. In addition, the preoperative axillary fixed lymphadenopathies were determined to have receded on physical examination and USG imaging, and the lymph nodes were observed to have replaced the thin cortex with a hyperechogenic fatty hilus (Figure 3).

In the histopathological examination of the specimen, a Grade 3 (Scarf Bloom Richardson) invasive ductal carcinoma $11 \mathrm{~cm}$ in diameter was determined. There were perineural and lymphovascular invasions, many satellite tumor nodules around the tumor that had led to ulceration and an appearance of infiltration to the epidermis and breast head/areola, continuation of the tumor with a deep surgical border, and infiltration to the striated muscle in this area. ER was determined as $80 \%, \mathrm{PR}$ as $5 \%$ positive and c-erb B- 2 as negative. In the postoperative period, aromatase inhibitor was administered. The results of consecutive complete blood tests showed that the NLR and PLR values of 7.05 (N: 84\%, L:1 1.9\%), and 407.5 (P: 326, L: $\left.0.8 \times 10^{9} / \mathrm{L}\right)$, respectively, on preoperative Day 1 decreased to 4.35 (N: 74\%, L: $17 \%)$ and 380 (P: $\left.266, \mathrm{~L}: 0.7 \times 10^{9} / \mathrm{L}\right)$, respectively, on postoperative Day 5 and to 2.63 (N: $65.2 \%, \mathrm{~L}: 24.7 \%)$ and 251 (P: 277, L: $\left.1.1 \times 10^{9} / \mathrm{L}\right)$, respectively, on postoperative Day 14 .

Written informed consent was obtained from the patient who participated in this case.

\section{DISCUSSION}

In cancer patients, the clinical prognosis is as much related to the properties of the tumor as to the patient's response to the tumor (5). In recent studies, cancer and immunology have become increasingly important. For many years, immunologists have stated that the immune response is suppressed in cancer patients and the response to the tumor is reduced (6). Especially in patients with excessive tumor loads, there is evident immune suppression; with reduction of the tumor load with primary surgery, the immune response has been seen to increase. This is explained by changes occurring in T-cells including increase of antitumor responses with the reduction in tumor load (4).

Neutrophil-to-lymphocyte ratio, which can be obtained from a peripheral full blood count, is a routine clinical marker of systemic inflammatory response $(2,3)$. Increased peripheral neutrophils before treatment and decreased lymphocyte count have been shown to have negative effects on the survival of cancer patients (7). In addition, it has been found that just as increased neutrophil numbers are related to the paraneoplastic activity of the tumor, reduced lymphocyte numbers are related to suppression of the immune system. While neutrophils, which affect tumor growth, are the main source of angiogenesis and growth factors, lymphocytes form the patient immune response with cytotoxic cell death and production of cytotoxin, which prevent proliferation of tumor cells (8). Increased NLR in breast cancer has been shown to have increased mortality (2). In addition, in a recent study, high NLR in breast cancer patients was found to be related to lymph node metastasis (3).

Although the effects of NLR on survival in breast cancer have been widely accepted as a prognostic factor, there are few studies supporting the use of PLR as an independent prognostic factor. However, experimental studies and clinical data have shown that platelet activation is a feature of cancer, with support of neoangiogenesis, destruction of the extracellular 
matrix, and expression of adhesion molecules and growth factors (9). Seeretis et al. (10) determined a relationship between increased PLR and metastatic lymph nodes in cancer patients.

In the case presented here, the tumor load was reduced with a simple mastectomy. When the blood values of the patient were examined during this procedure, a continuous reduction in NLR was recorded together with the reduction in tumor load. The regression observed in the masses in the chest wall of the patient can be related to the decrease in the NLR value. Even though no absolute relationship has yet been shown between PLR and breast cancer, the regression of the tumor in this case together with decreased PLR values suggests that PLR can be effective in tumor prognosis.

\section{CONCLUSION}

In patients with locally advanced breast cancer, decreases in NLR and PLR values parallel to the reduction of tumor load with surgery may cause an increase in immune response and regression of the tumor. Further studies with an extensive series are required to better understand this relationship.

Informed Consent: Written informed consent was obtained from patient who participated in this study.

Peer-review: Externally peer-reviewed.

Author Contributions: Concept - A.D., A.E.; Design - A.D., G.D., A.E.; Supervision - A.E.; Resource A.D., A.E.; Materials - A.D., A.E.; Data Collection and/or Processing - A.D., G.D., A.E.; Analysis and/or Interpretation - A.D., G.D., A.E.; Literature Search - A.D., G.D., A.E.; Writing Manuscript - A.D., G.D., A.E.; Critical Reviews - A.D., G.D., A.E.

Conflict of Interest: The authors have no conflicts of interest to declare.

Financial Disclosure: The authors declared that this study has received no financial support.

\section{REFERENCES}

1. Sinacki $M$, Badzio A, Welnicka-Jaskiewicz M, Bogaerts J, Piccart MJ, Therasse $P$, et al. Pattern of care in locally advanced breast cancer: focus on local therapy. Breast 2011; 20: 145-150. [CrossRef]

2. Azab B, Bhatt VR, Phookan J, Murukutla S, Kohn N, Terjanian T, et al. Usefulness of the neutrophil-to-lymphocyte ratio in predicting short- and long-term mortality in breast cancer patients. Ann Surg Oncol 2012; 19: 217-224. [CrossRef]

3. Eroğlu A, Akbulut $S$. The role of pretreatment neutrophil to lymphocyte ratio and platelet to lymphocyte ratio in the diagnosis of breast cancer: predicting lymph node metastasis. World Oncol 2013; 4: 262-263.

4. Salvadori S, Martinelli G, Zier K. Resection of solid tumors reverses T cell defects and restores protective immunity. Journal Immunol 2000; 164: 2214-2220. [CrossRef]

5. Roxburgh CS, McMillan DC. Role of systemic inflammatory response in predicting survival in patients with primary operable cancer. Future Oncol 2010; 6: 149-163. [CrossRef]

6. Schreiber H. Tumor immunology. In: Paul WE, editor. Fundamental Immunology. 4th ed Philadelphia: Lippincott \& Raven; 1999. p.1237-1270.

7. Schmidt H, Suciu S, Punt CJ, Gore M, Kruit W, Patel P, et al. Pretreatment levels of peripheral neutrophils and leukocytes as independent predictors of overall survival in patients with American Joint Committee on Cancer Stage IV Melanoma: results of the EORTC 18951 Biochemotherapy Trial. J Clin Oncol 2007; 25: 1562-1569. [CrossRef]

8. Strieter RM, Burdick MD, Mestas J, Gomperts B, Keane MP, Belperio JA. Cancer CXC chemokine networks and tumour angiogenesis. Eur J Cancer 2006; 42: 768-778. [CrossRef]

9. Kono SA, Heasley LE, Doebele RC, Camidge DR. Adding to the mix: fibroblast growth factor and platelet-derived growth factor receptor pathways as targets in non-small cell lung cancer. Curr Cancer Drug Targets 2012; 12: 107-123. [CrossRef]

10. Seretis C, Seretis F, Lagoudianakis E, Politou M, Gemenetzis G, Salemis NS. Enhancing the accuracy of platelet to lymphocyte ratio after adjustment for large platelet count: a pilot study in breast cancer patients. Int J Surg Oncol 2012; 2012: 653608. [CrossRef] 